\title{
A Comparative Picture of Structural and Electrochemical Properties of Fluorinated Liquid Crystals - a Theoretical Study
}

\author{
S. PRASAD AND D.P. OJHA* \\ School of Physics, Sambalpur University, Jyoti Vihar-768 019, Sambalpur, Odisha, India \\ (Received March 24, 2017; in final form July 5, 2017)
}

\begin{abstract}
A comparative picture of structural and electrochemical properties of fluorinated liquid crystals viz. $\quad p$-phenylene-4-methoxybenzoate-4-trifluoromethylbenzoate (FLUORO1), and 4-propyloxyphenyl-4-(4trifluoromethyl benzoyloxy) benzoate (FLUORO2) have been studied. The structure of liquid crystals have been optimized by the density functional B3LYP with 6-31 $+\mathrm{G}(\mathrm{d})$ basis set using crystallographic geometry as input. Molecular charge distribution, and phase stability of these molecules have been analyzed based on the Mulliken, and Loewdin population analysis. The electronic absorption spectra of molecules have been simulated by employing the density functional theory method. The effects of substituted groups on electrochemical properties such as highly occupied molecular orbital, lowly unoccupied molecular orbital energies, and energy gap $\left(E_{g}\right)$ have been investigated. It has been observed that substitution of additional alkyl groups leads to prominent decrement in electrochemical properties.
\end{abstract}

DOI: 10.12693/APhysPolA.132.1383

PACS/topics: HOMO, LUMO, phase stability, oscillator strength

\section{Introduction}

Liquid crystals (LCs) are an enthralling set of soft condensed matter characterized by the insightful amalgamation of long-range order, and fluidity. The unique subtle balance between order and fluidity is not only deeply fascinating from a scientific point of view, but it gives rise to a broad range of spectacular spectral phenomena that are far from fully explored [1].

The LCs were also used as carrier materials in the examination of nonlinear optical properties/parameters (e.g., nonlinear refractive index and nonlinear optical absorption) [2] of organic molecules. For the active matrix LCD applications, such as thin-film-transistor (TFT), and metal-insulator-metal (MIM), "fluoro" is the preferred polar group as it exhibits a high resistivity, low viscosity, and low birefringence [3]. It gives excellent chemical and thermal stability because of the strong, and unique physical properties of the $\mathrm{C}-\mathrm{F}$ bond, which contributes to the display, and photonic applications. There are many good reasons to make use of the unique properties of fluorinated structures for design of LCs, and these far outweigh the economic and synthetic disadvantages. Although most of the fluoro based LCs have a strong tendency to form smectic phases [4], they have become indispensable as components of LC mixtures for all kinds of reflective or transflective LC display for battery-powered devices with low energy consumption. Further, the lightinduced property changes in LCs [5] enable fabrication of suitable materials for dynamic holography, which opens a way for construction of all-optical light modulators,

*corresponding author; e-mail: durga_ojha@hotmail.com2 phase conjugate mirrors, optical amplifiers, optical correlators etc. [6].

The methods based on time dependent density functional theory (TDDFT) applied to small and middle sized systems $[7,8]$ provide rather good accuracy at low computational cost. These methods still remain of rather limited application for establishing realistic molecular models, and biological systems.

In the present article, the quest is towards the molecular design of fluorine based low absorption liquid crystals for possible applications [9]. A comparative picture of structural and electrochemical properties of FLUORO1, and FLUORO2 molecules have been presented. In particular, it concentrates on the alternation of these properties with respect to substituent effect. The highly occupied molecular orbital (HOMO, H), lowly unoccupied molecular orbital (LUMO, L) energies, and energy gap $\left(E_{g}\right)$ have also been reported. An examination of thermodynamic data has revealed that FLUORO1 has a nematicisotropic transition at $506 \mathrm{~K}$, and FLUORO2 shows a smectic A-isotropic transition temperature at $488 \mathrm{~K}$ [10].

\section{Theoretical approach}

The main difficulties against a reliable theoretical approach are concerned with the size of such systems, and the presence of strong electron correlation effects. Both properties are difficult to treat in the framework of the quantum mechanical methods rooted in the HartreeFock (HF) theory. Density functional theory (DFT) is successful to evaluate a variety of ground-state properties with accuracy close to that of post-HF methods $[9,11]$. In this context, remarkable structural predictions have been obtained especially using the "hybrid" density functionals [12-14] such as B3LYP combining "exact exchange" with gradient-corrected density functionals. As a consequence, there is currently a great interest in extending 
DFT to excited electronic states. The TDDFT approach offers a rigorous route to the calculation of vertical electronic excitation energies, and other spectral characteristics $[15,16]$.

The present molecular models comprise of the fluorine based liquid crystals FLUORO1 and FLUORO2. The geometries optimizations have been performed using the DFT approach [9], and in particular the Becke3Lee-Yang-Parr hybrid functional (B3LYP) exchangecorrelation functional, and the $6-31 \mathrm{G}+(\mathrm{d})$ basis set. The DFT approach was originally developed by Hohenberg and Kohn [17], Kohn and Sham [18], and Jones and Gunnarson [19] to provide an efficient method of handling the many-electron system. The theory allows us to reduce the problem of an interacting many-electron system to an effective single-electron problem. The DFT calculations have been performed by a spectroscopy oriented configuration interaction procedure (SORCI) [20].

The general structural parameters of the systems such as bond lengths and bond angles have been taken from the published crystallographic data [10] to construct the electronic structures. The charge distributions of molecules have been calculated by performing Mulliken, and Loewdin population analysis. The mesomorphic behavior and the nematic phase stability have been predicted through the calculated local charge distributions.

\section{Results and discussion}

The electronic structures of FLUORO1 and FLUORO2 have been shown in Fig. 1. Each ester linkage is coplanar with the benzene ring to which the $\mathrm{C}$ atom of the linkage is attached [10]. The group charges, electronic absorption spectra, and phase stability of the molecules have been discussed below.
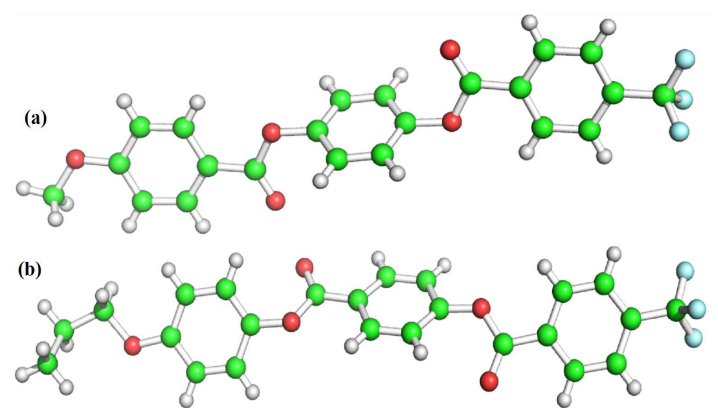

Fig. 1. The geometric structures of (a) FLUORO1, and (b) FLUORO2 molecules.

\subsection{Group charges}

The group charges are needed to explain the phase behavior, and transition temperatures of mesogens. The Mulliken population analysis has been performed that partitions the total charge among the atoms in the molecule, and compared with the Loewdin population analysis results. However, there is much agreement between the methods while it comes to the group charges of each molecule. It is evident from Table I that the positively charged alkyl chains of FLUORO1 will be strongly attracted by the negatively charged side group as well as the core, causing the formation of longer units in the nematic phase. Hence, the phase stability is expected to be high for FLUORO1. Further, the thermal vibration amplitude of the end chain carbon atoms in FLUORO2 increase markedly with the increase of chain length, indicating a low packing efficiency. This leads to the drastic decrease in phase stability, and transition temperature. This supports the transition temperatures reported by the crystallographer (Table I).

TABLE I

Mulliken M and Loewdin L group charges and nematicisotropic transition temperatures [10] for FLUORO1, and FLUORO2 molecules.

\begin{tabular}{c|c|c|c|c|c|c}
\hline \hline \multicolumn{2}{c|}{ Core } & \multicolumn{2}{c|}{ Side group } & \multicolumn{2}{c|}{ End group } & \multirow{2}{*}{ T [K] } \\
\cline { 1 - 4 } $\mathrm{M}$ & $\mathrm{L}$ & $\mathrm{M}$ & $\mathrm{L}$ & $\mathrm{M}$ & $\mathrm{L}$ & \\
\hline \multicolumn{7}{c}{ FLUORO1 } \\
\hline-0.21 & -0.14 & -0.16 & -0.13 & 0.37 & 0.26 & 506 \\
\hline \multicolumn{7}{c}{ FLUORO2 } \\
\hline-0.45 & -0.29 & -0.14 & -0.12 & 0.59 & 0.40 & 488
\end{tabular}

\subsection{Electronic absorption spectra}

The electronic absorption spectrum of FLUORO1 is shown in Fig. 2. In the UV region, three strong absorptions at $211.72 \mathrm{~nm}\left(\lambda_{1}\right), 245.12 \mathrm{~nm}\left(\lambda_{2}\right)$, and $267.97 \mathrm{~nm}$ $\left(\lambda_{3}\right)$ have been observed. However, no absorption has been observed in the visible region. The strongest band appears in a region of $204.10 \mathrm{~nm}$ to $227.54 \mathrm{~nm}$ with absorption maxima $\left(\lambda_{\max }\right)$ at $211.72 \mathrm{~nm}\left(\lambda_{1}\right)$. This band arises from the $\mathrm{HOMO} \rightarrow \mathrm{LUMO}$ transition, and is assigned as $\pi \rightarrow \pi^{*}$ transition in the molecule. Further, the calculation also predicts $\pi \rightarrow \pi^{*}$ transitions corresponding to weak absorption bands corresponding to two wavelengths $\lambda_{2}$ and $\lambda_{3}$, respectively.

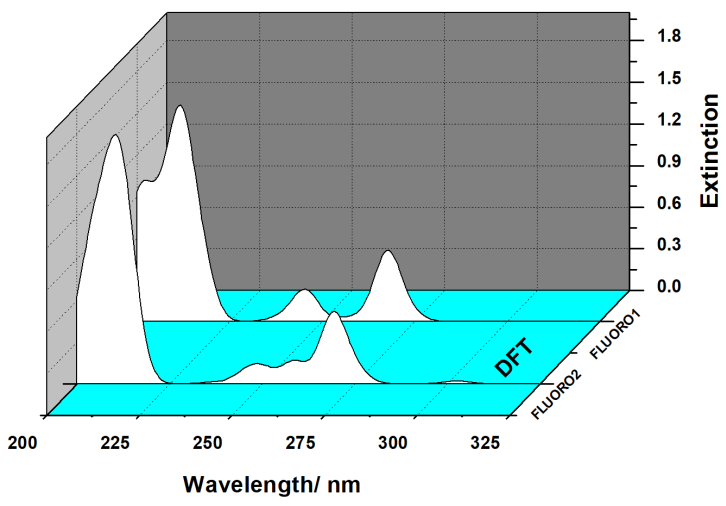

Fig. 2. Electronic absorption spectra of FLUORO molecules using TDDFT approximation. Extinction unit: $10^{4} \mathrm{dm}^{3} \mathrm{~mol}^{-1} \mathrm{~cm}^{-1}$.

Figure 2 also shows the three prominent bands in the UV region for FLUORO2 with absorption maxima at $210.55 \mathrm{~nm}\left(\lambda_{1}\right), 248.63 \mathrm{~nm}\left(\lambda_{2}\right)$, and $269.14 \mathrm{~nm}\left(\lambda_{3}\right)$. The strongest band has been observed from $200 \mathrm{~nm}$ to 
$225 \mathrm{~nm}$ with absorption maxima $\left(\lambda_{\max }\right)$ at $210.55 \mathrm{~nm}$. This band arises due to the HOMO $\rightarrow$ LUMO transition and is assigned as $\pi \rightarrow \pi^{*}$ transition in the molecule. However, the other weak absorption bands at $\lambda_{2}$, and $\lambda_{3}$ also indicate the possibility of $\pi \rightarrow \pi^{*}$ transitions. The strongest absorption band for both the molecules has been found almost in the same range of wavelength (FLUORO1: 204.10-227.54 nm and FLUORO2: 200$225 \mathrm{~nm}$ ). This indicates that the absorption spectrum of both molecules in the UV region are identical because of the identical $\pi$-electron structure.

\subsection{Oscillator strength}

The oscillator strength is a dimensionless quantity that expresses the probability of absorption of electromagnetic radiation in transitions between energy levels of an atom or molecule. It indicates the allowedness of electronic transitions in a molecule, and it is particularly valuable as a method of comparing "transition strengths" between different types of quantum mechanical systems. The wavelength dependence of oscillator strength corresponding to UV absorption spectrum using the DFT data has been shown in Fig. 3. The substitution of additional alkyl groups drastically changes the oscillator strength value. Further, the oscillator strength remains almost constant in a particular UV wavelength region for FLUORO1 molecule (Fig. 3), which may be exploited for possible applications. FLUORO2 molecule does not show any consistency of oscillator strength as a function of wavelength. Evidently, the FLUORO1 molecule exhibits good consistency in oscillator strength as a function of wavelength. Further, the consideration of maximum oscillator strength value indicates that the FLUORO2 molecule exhibits higher oscillator strength at longer wavelength compared to FLUORO1. Hence, FLUORO2 is much flexible for electronic transitions, and its photostability is high.

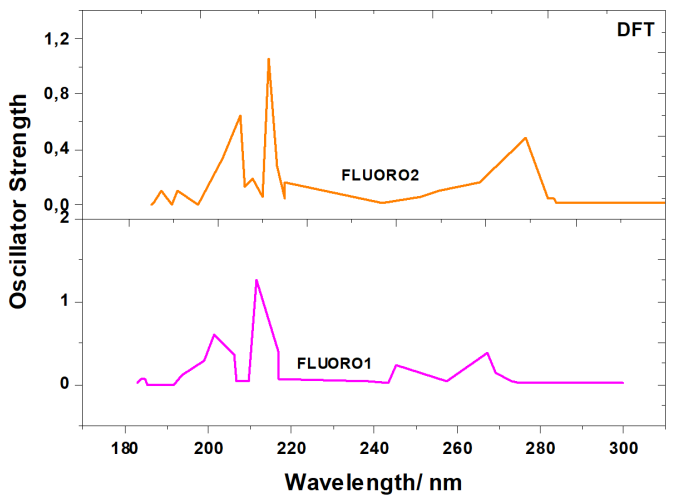

Fig. 3. Wavelength dependence of oscillator strength for FLUORO molecules.

\subsection{Electrochemical properties}

A comparative picture of electrochemical properties such as HOMO (H), LUMO (L) energies, and energy gap $\left(E_{g}\right)$ of FLUORO1 and FLUORO2 molecules have been presented in Table II. The energy gap between the HOMO and LUMO is an important parameter in determining molecular electrical transport properties, and photostability. It is also a critical parameter in determining the molecular electrical transport properties as it is a measure of electron conductivity. The substitution of additional alkyl groups leads to a prominent decrement in HOMO $(\mathrm{H})$, LUMO $(\mathrm{L})$ energies, and energy gap $\left(E_{g}\right)$ values. The FLUORO2 molecule exhibits a lower energy gap; hence its electron conductivity is high. Lower value of the energy gap explains the eventual charge transfer interactions taking place within the molecule.

TABLE II

The HOMO H, LUMO L energies, and energy gap $E_{g}[\mathrm{eV}]$ of FLUORO molecules at TDDFT levels.

\begin{tabular}{c|c|c|c}
\hline \hline Method & HOMO & LUMO & $E_{g}=E_{L}-E_{H}$ \\
\hline \multicolumn{4}{|c}{ FLUORO1 } \\
\hline DFT & -8.80 & -0.98 & 7.82 \\
\hline \multicolumn{3}{c}{ FLUORO2 } \\
\hline DFT & -8.47 & -1.23 & 7.24
\end{tabular}

\section{Conclusions}

The present computations lead to the following conclusions:

1. The substitution of additional alkyl groups lead to a prominent decrement in $\operatorname{HOMO}(\mathrm{H}), \mathrm{LUMO}(\mathrm{L})$ energies, and energy gap $\left(E_{g}\right)$ values. The FLUORO2 molecule exhibits a lower energy gap; hence its electron conductivity is high.

2. The both molecules show strong absorption bands in UV range. Further, the strongest bands appear almost in the same range of wavelength because of the identical $\pi$-electron structure.

3. FLUORO1 molecule exhibits good consistency in oscillator strength as a function of wavelength. Further, the consideration of maximum oscillator strength value indicates that the FLUORO2 molecule exhibits higher oscillator strength at longer wavelength compared to FLUORO1. Hence, FLUORO2 is much flexible for electronic transitions, and its photostability is high.

4. These studies provide valuable information to predict their nonlinear optical properties/parameters e.g., nonlinear refractive index and nonlinear optical absorption. The present article provides a new and interesting way to address this problem.

\section{Acknowledgments}

One of the authors, Seema Prasad is thankful to the UGC, New Delhi, India for providing financial support as JRF under RGNF scheme. 


\section{References}

[1] A. Miniewicz, J. Mysliwiec, F. Kajzar, J. Parka, Opt. Mater. 28, 1389 (2006).

[2] S. Nagappa, J. Mahadeva, R. Somashekar, C.V. Yalemaggad, G. Umesh, K.B. Manjunatha, Mol. Cryst. Liq. Cryst. 540, 88 (2011).

[3] P. Kirsch, Modern Fluoro Organic Chemistry, WileyVCH, Germany 2004.

[4] M. Hird, Chem. Soc. Rev. 36, 2070 (2007).

[5] Y. Wu, Y. Zhou, L. Yin, G. Zou, Q, Zhang, Liq. Cryst. 40, 726 (2013).

[6] R. Eelkema, Liq. Cryst. 38, 1641 (2011).

[7] M.E. Castro, M.J. Percino, V.M. Chapela, G.S. Moro, M. Ceron, F.J. Melendez, J. Mol. Model. 19, 2015 (2013).

[8] W. Koch, M.C. Holthausen, A Chemist's Guide to Density Functional Theory, Wiley-VCH, Weinheim 2000.

[9] Y. Chen, H. Xianyu, J. Sun, P. Kula, R. Dabrowski, S. Tripathi, R.J. Twieg, S.T. Wu, Opt. Exp. 19 10843 (2011).

[10] K. Hori, C. Kubo, H. Okamoto, S. Takenaka, Mol. Cryst. Liq. Cryst. 365, 617 (2001).
[11] A. Neugebauer, G. Hafelinge, J. Mol. Struct. Theochem. 585, 35 (2002).

[12] K. Sharkas, J. Toulouse, A. Savin, J. Chem. Phys. 134, 064113 (2011).

[13] K. Sharkas, A. Savin, H.J.A. Jensen, J. Toulouse, J. Chem. Phys. 137, 044104 (2012).

[14] Y. Cornaton, O. Franck, A.M. Teale, E. Fromager, Mol. Phys. 111, 1275 (2013).

[15] P.L. Praveen, D.P. Ojha, Phys. Rev. E 83, 051710 (2011).

[16] S. Sergeyev, E. Pouzet, O. Debever, J. Levin, J. Gierschner, J. Cornil, R.G. Aspe, Y.H. Geerts, J. Mater. Chem. 17, 1777 (2007).

[17] P. Hohenberg, W. Kohn, Phys. Rev. 136, B864 (1965).

[18] W. Kohn, L.J. Sham, Phys. Rev. 140, A1133 (1965).

[19] R.O. Jones, O. Gunnarsson, Rev. Mod. Phys. 61, 689 (1989).

[20] F.A. Neese, J. Chem. Phys. 119, 9428 (2003). 